\title{
Comparative Structures of 3,4-Dichlorophenol and 3,4-Dichlorophenolate
}

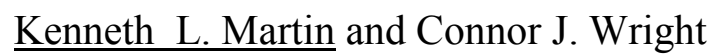

Department of Chemistry \& Biochemistry, Berry College, Mount Berry, Georgia 30149

Crystals were obtained from a solution containing 3,4-dichlorophenol and imidazole in 1:1 stoichiometric proportions. These are of a $2: 1$ co-crystal $[P-1, a=7.6731(7) \AA, b=8.2007(7) \AA$, $\left.c=14.229(2) \AA, \alpha=80.320(1)^{\circ}, \beta=84.070(1)^{\circ}, \gamma=68.699(1)^{\circ}\right]$ of 3,4-dichlorophenol and imidazole. One of the phenol molecules donates a hydrogen bond to the imidazole molecule and is a hydrogen bond acceptor from the second phenol. A second reaction mixture (in which equal moles of 3,4-dichlorophenol and tetramethylammonium hydroxide were added together) resulted in other crystals. These are of a $2: 1$ co-crystal $\left[P 2_{1} / n, a=10.3392(4) \AA, b=16.6235(5) \AA, c=\right.$ 14.7074(5) $\AA, \beta=91.602(3)^{\circ}$ ] of 3,4-dichlorophenol and tetramethylammonium 3,4dichlorophenolate. In these crystals, one of the phenols is disordered while the second phenol molecule is not, and the phenolate is accepting hydrogen bonds from two phenol molecules. The structures of the 3,4-dichlorophenol(ate) moieties will be compared to each other and to that of pure 3,4-dichlorophenol. 